\title{
Machine Learning and Artificial Intelligence Based Identification of Risk Factors and Incidence of Gastroesophageal Reflux Disease in Pakistan
}

\author{
Mustafa Kamal Pasha \\ Department of Environment, Society and Design, Lincoln University - New Zealand \\ E-mail: kpasha2003@gmail.com
}

Received: 04 December 2020; Accepted: 25 January 2021; Published: 08 October 2021

\begin{abstract}
The disease burden of Gastroesophageal Reflux Disease (GERD) varies across the globe and have a significant impact on the overall health of the communities. A number of complications and diseases stem from chronic GERD. In order to provide improved healthcare measures and to effectively monitor and control GERD, it is important to identify rate of incidence of the disease and the associated risk factors along with symptoms. Therefore, this study was conducted by retrieving the relevant data through machine learning. Principles of Artificial Neural Networks were applied to sort the data and the results were obtained in the form of a network by using VOSviewer software. These artificial intelligence and machine learning based results reveal that the Asian population is increasingly becoming prone to GERD and sporadic reports from Pakistan have surmounted to disclose that GERD is constantly present across different districts and cities of Pakistan. The major risk factors identified among the Pakistani population in different research articles include consumption of oily foods, the habit of having late dinners, sedentary lifestyles and a lack of understanding about disease diagnosis, and GERD management and treatment. Our results suggest that acid reflux and inflammation of esophageal cavity are some of the main symptoms of the disease. On the basis of the results obtained, it is speculated that this study will provide a ground to improve the symptomatic diagnosis of GERD by closely observing and analyzing the risk factors and the rate of incidence with symptoms. It would enable the healthcare facilities to effectively monitor the GERD cases so that the disease burden due to GERD and related illnesses could be reduced. Moreover, the identification of regional differences and a comparative data would help us in identifying the disease hotspots where more efforts would be needed to manage and control the disease.
\end{abstract}

Index Terms: Gastroesophageal reflux disease, GERD, Pakistan, risk factors, prevalence, artificial intelligence, machine learning

\section{Introduction}

Gastroesophageal reflux disease or GERD is a commonly reported disease with a worldwide prevalence. According to Argyrou et al., 2018, around 10-20\% cases are present on a constant basis in the western world, while the East has varying percentages of the disease in different regions. Pakistan has been reported to have around $22 \%$ of GERD prevalence, as per reports from patients presenting to different clinics and hospitals across the country. The disease burden has been reported to have followed an increasing trend in the West as well as in Asia. It not only affects the quality of a patient's life, but also the economic burden which concerns the health sector. There are various definitions of GERD found in the data pool, which all lead to the understanding that it is a condition where stomach reflux lead to severe consequences such as acidity, heart burn, injury to the esophagus and cough. The symptoms of GERD also relate to the same understanding and include heartburn and reflux of acid towards the esophagus and sometimes even towards the oral cavity. These symptoms are further associated with injury or damage to the esophagus and inflammation of tissues across the esophagus (Argyrou et al., 2018). Furthermore, according to Veugelers et al., 2006, untreated cases or improper disease management can lead to severe consequences including, anemia, weight loss, dysphagia and in worst cases, adenocarcinoma (Veugelers et al., 2006). Therefore, it is important to take control measures to effectively manage the disease.

The foremost step in disease management is to identify the disease symptoms and the risk factors so that enough knowledge would be available to avoid any misdiagnosis. Moreover, a better diagnosis of disease will also enable us to 
identify the disease hotspots where more healthcare facilities would be required. As the disease prevalence is high and there is a lack of awareness of GERD in population, so, the knowledge about the disease basics must be reviewed and should be made available to population through healthcare providers and media. Therefore, this study was conducted to utilize the principles of artificial intelligence to sort out the huge volumes of data in order to effectively tackle the disease. As according to Munawar et al., 2020 the increased use of computers and internet sources is the ideal way to collect vast volumes of data (Munawar et al., 2019a; Munawar et al., 2019b; Munawar).Therefore, the approach of artificial intelligence and machine learning will be utilized in this study to answer all the questions about GERD, including a detailed analysis of the disease pathophysiology, symptoms, treatment options and prevalence among the Pakistani population, with a special focus on comparing the incidence of GERD between the populations of different cities of Pakistan. Now a days the devices like antennas and wireless devices are also being used for the collection of scientific data. These devices would also have larger benefits, such as wearable antennas are now being used in personal wellbeing i.e. disease treatments etc (Munawar; Munawar et al., 2017; Munawar; Munawar and Maqsood) However, for our research article we preferred artificial intelligence which will include data collection through wireless devices. The methodology adopted in this research to collect and analyse the data related to GERD is described in detail.

\section{Methodology}

The methodology adopted for this research is given in Fig. 1 while Fig. 2 represents the framework of this study.

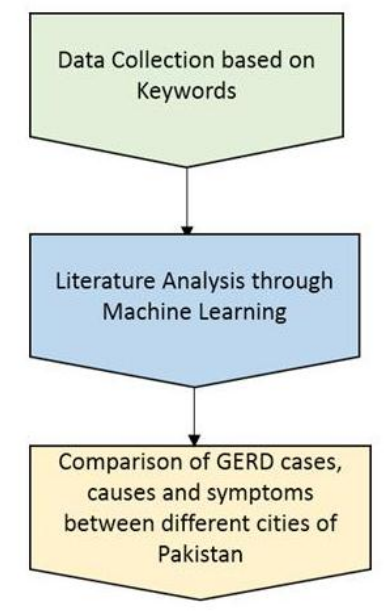

Fig. 1 AI based methodology adopted for the identification of risk factors of GERD disease

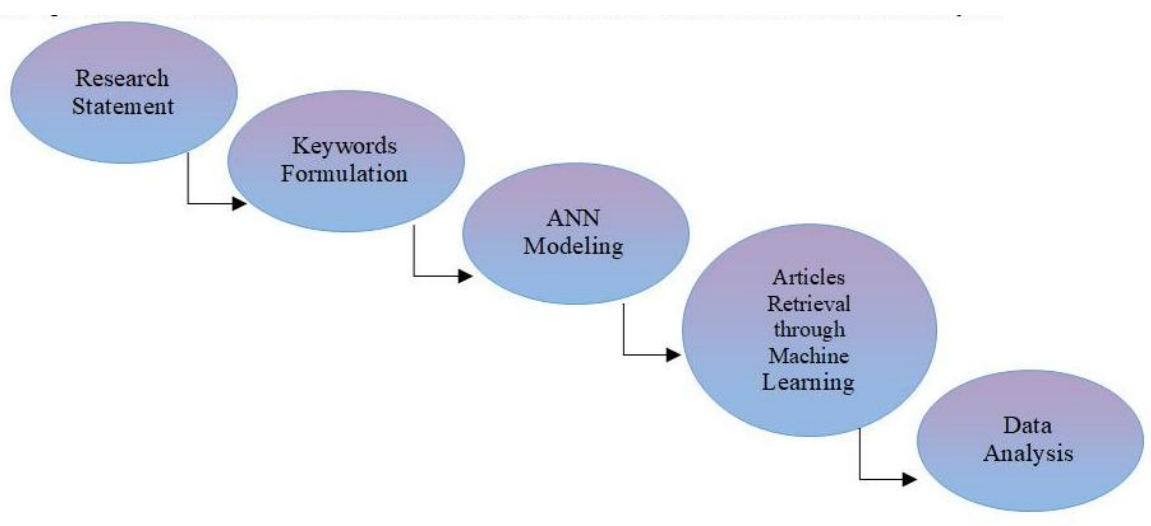

Fig. 2 Framework of Research

We started this study by identifying the exploratory domain on the basis of which research question was formulated. Relevant data was retrieved and was subjected to artificial intelligence based data processing. The output data obtained was visualized by using VOSviewer software and was further subjected to analysis.

\section{1 Data Retrieval}

A combination of keywords was generated on the basis of which, data was retrieved by using machine learning. The keywords used for this research includes, Gastroesophageal reflux disease", "GERD in Pakistan", "Risk factors of 
GERD”, "Prevalence of GERD". All of these keywords are used for the detailed analysis of the study through artificial intelligence approach. Based on these keywords, literature was selected from different search engines including Web of Science, Scopus and ScienceDirect. High index research papers were selected from top-tier journals. After selection of the multiple sets of articles, different keyword queries were formulated and to make links between them, neuron links were established via ANN modelling.

\section{2 ANN Modeling}

Multilayer Artificial Neural Networks are used in three layers that are shown in Fig 3. as input layer, hidden layer and output layer to collect and process the complex data. The data retrieved through different search engines is fed into the input layer. Input layer form links with hidden layer where the data is processed. After this processing, it is obtained from output layer. VOSviewer software is used to visualize the output data so that it could be interpreted. The aim of these complex networks is to find the weight of the data that will provide a descriptive picture of GERD disease. Using this ANN model all the data is collected and evaluated in this research.

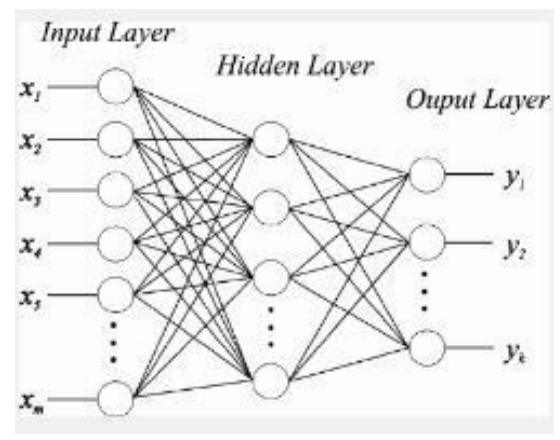

Fig. 3 ANN Model for the data collection

\section{Results}

\subsection{ANN Model}

The data from output layer of ANN model is visualized through VOSviewer software which constructs a map based on similarity among the data. It represents the data from main exploratory domains via nodes. The size of these nodes determine the weight of the data. Moreover, the lines between the nodes represent the interconnectedness of this data. It can be seen from Fig 4 that either the data obtained is from pathology domain or it is epidemiological data. The lines represent that how these both domains are linked which illustrates that for better disease control and monitoring, it is important to understand disease epidemiology with reference to its pathology.

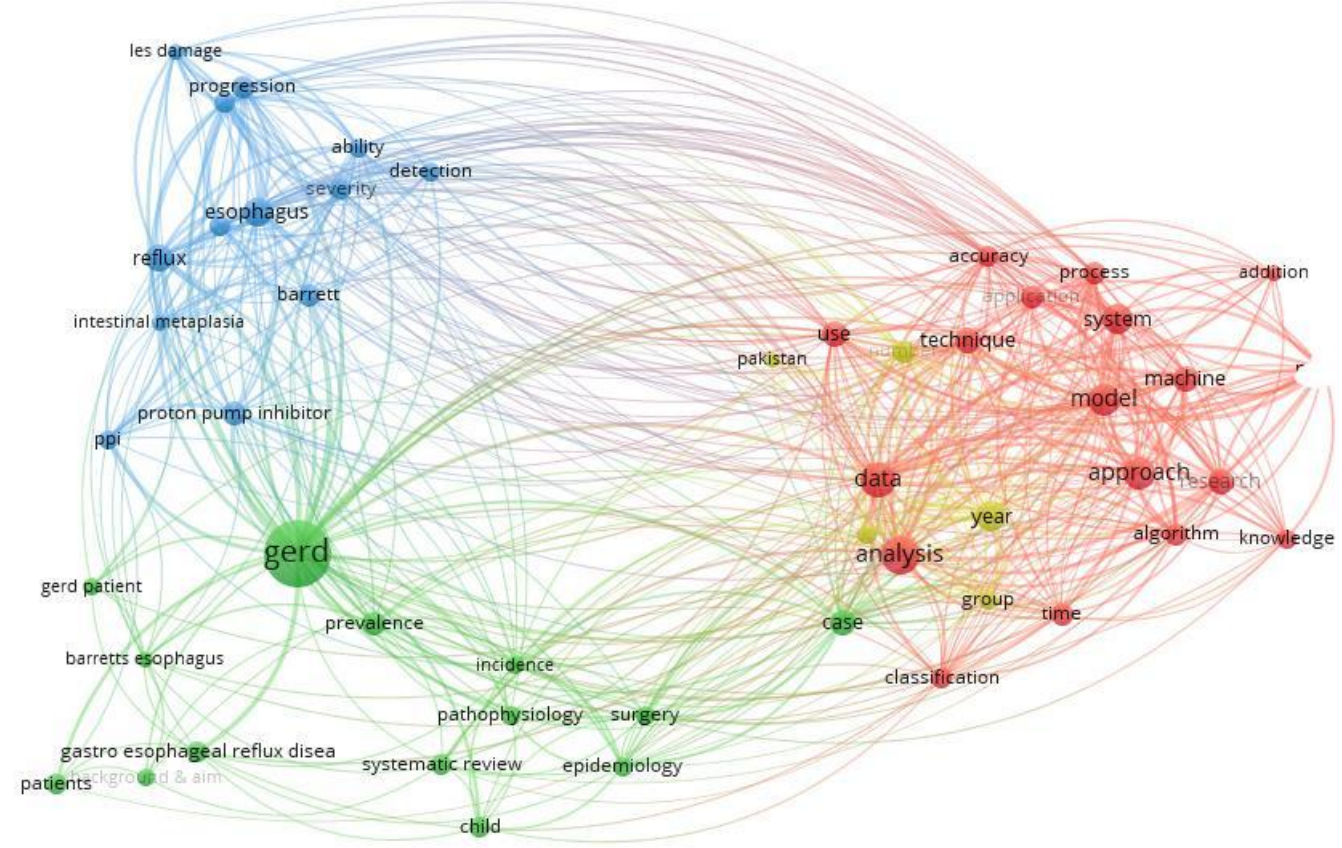

Fig. 4 Keywords found in literature related to GERD 
After identification of the importance of epidemiology, data on risk factors, symptoms and prevalence of GERD is obtained through machine learning and is further described below in detail with the future suggestions.

\subsection{Data Collected through Artificial Intelligence and Machine Learning related to GERD}

The approach of artificial intelligence and machine learning is utilized in this study to answer all the questions about the GERD including a detailed analysis of the disease pathophysiology, symptoms, treatment options and prevalence among the Pakistani population, with a special focus on comparing the incidence of GERD between the populations of different cities of Pakistan.

\subsubsection{Disease Pathophysiology}

According to Herbella \& Patti, 2010, the pathophysiology of GERD is complex and crucial to understand so as to develop a concrete means of identifying treatment options and ways to prevent severe injuries or complications of chronic GERD. The non-erosive form of GERD develops in the absence of mucosal damage and is due to injury of the esophagus by the acid reflux. The stomach secretions such as pepsin, bile and gastric acid penetrate into the basal layer of the mucosa, thereby, causing inflammation. Such manifestations are possible when the protective layers of the mucosa are damaged or injured. These protective layers include the epithelial defence layers, constituting three different layers of epithelium, namely; pre-epithelium, the middle epithelial layer and the post-epithelial layer. Any damage in these layers due to ionic imbalance, excessively high acid concentration and reduced secretion of protective substance such as mucin, epidermal growth factor and prostaglandin E2 leads to the development of GERD. A detailed overview of pathogenesis of GERD is shown in Fig 5 which shows that how different causative agents contribute to GERD onset (Herbella \& Patti, 2010).

Gastroesophageal Reflux Disease (GERD): Pathogenesis and Clinical Findings

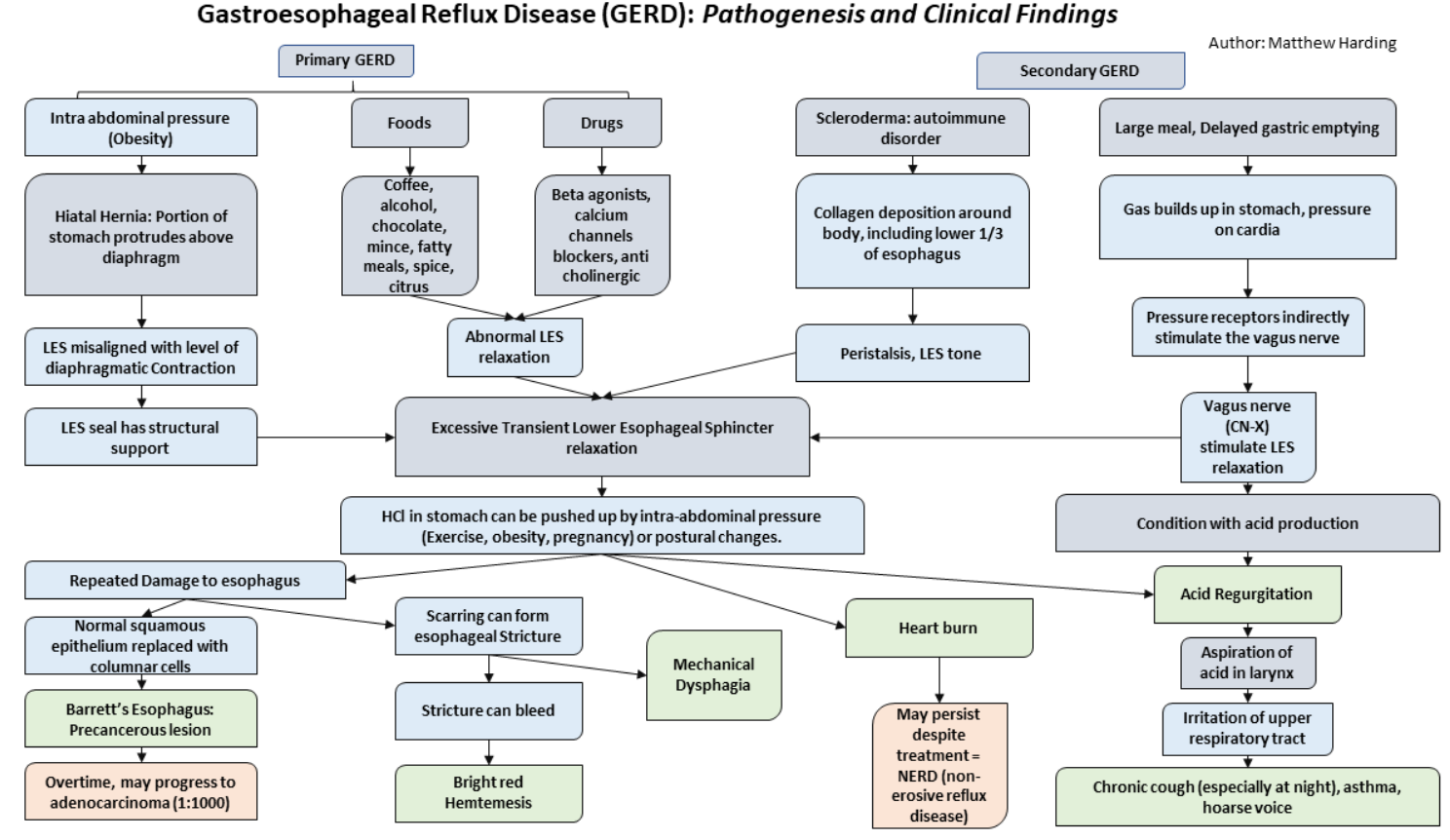

Fig. 5 Pathogenesis of GERD based on data collected through AI

Other factors conferring the pathophysiology of GERD highlighted by Menezes \& Herbella, 2017, include imbalanced pressures across the thoracic cavity, vulva and the diaphragm. According to The Menezes \& Herbella, 2017, basic mechanism which prevents GERD is known as the vulvar mechanism, which includes a negative pressure at the thorax to prevent uptake of acids coming upwards due to a positive pressure generating at the diaphragm. The overall structures and pressures present within this tract act to prevent refluxes. Injuries and damage to the pathway are involved in the generation of reflux and sending acids towards the esophagus. Thus, GERD is associated with a defective pressure gradient across the esophagus and gastric junctions (Menezes \& Herbella, 2017).

Mao et al., 2011, highlighted the association of esophageal cancer as one of the most alarming situations linked with GERD. Over the years, the association of adenocarcinoma of the esophagus has increasingly been associated with a prolonged history of GERD. According to Mao et al., 2011, the Asian countries, such as Japan, China and India have been reported to have a high incidence of esophageal adenocarcinoma. The western world also has an alarmingly increasing rate of esophageal adenocarcinoma. Almost 50\% of the worldwide cases of esophageal adenocarcinoma are reported from China alone. The progression of the disease is rapid, resulting in a high mortality rate (Mao et al., 2011). 
The percentage of disease vary in all the ages as shown in Fig.6. It is evident from Fig.6 that more than half of the 4-6 months old infants experience regurgitation more than one time per day. Occurences of regurgitations reduce after this age and then it is replaced by heartburn sensation in children and adults.

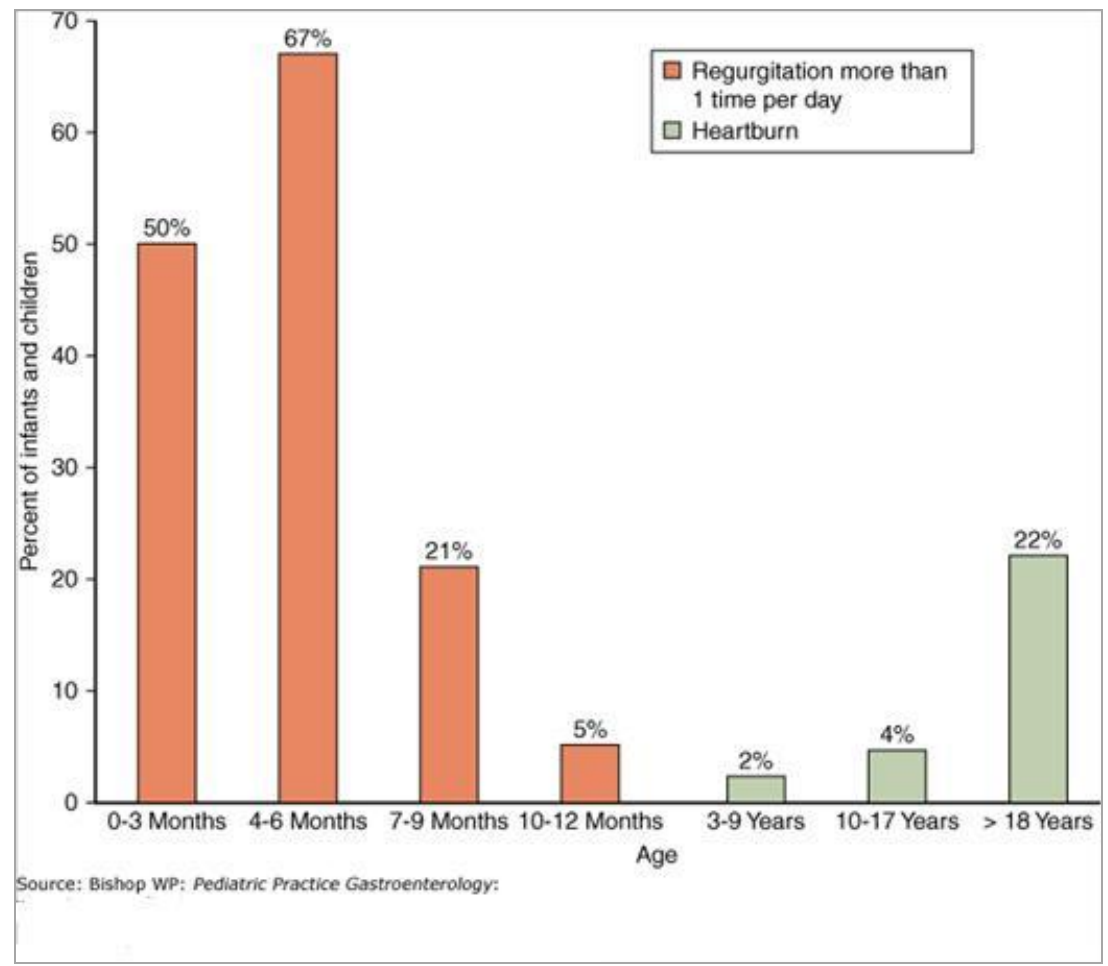

Fig. 6 Prevalence of disease in different age groups

\subsubsection{Possible causes of GERD}

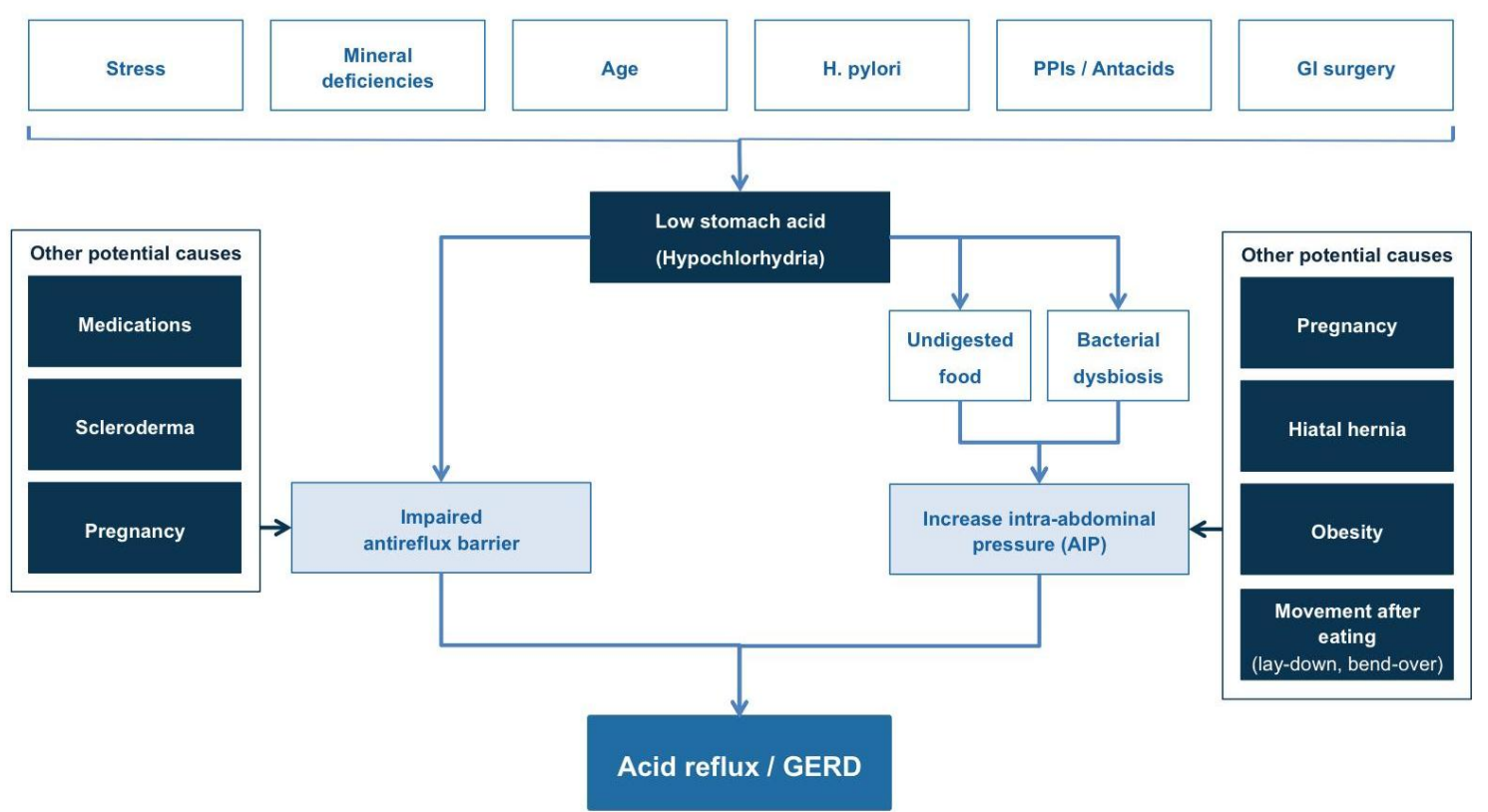

Fig. 7 Causes of GERD found in the data pool

Studies on lifestyle and eating habits have revealed a strong link of these factors with the development and progression of GERD. The Pakistani population has been deemed as prone to GERD due to various lifestyle habits such as late meals, oil and fat rich foods, small ratio of time between dinner and bedtime and an overall sedentary lifestyle (Fujiwara et al., 2005), smoking, increasing age and cultural background, as shown in Fig 7. It was found that the habit 
of walking post dinner significantly reduced the acid reflux, thereby reducing the chances of developing GERD (Karim et al., 2011). The high rate of Helicobacter pylori infections in the Asian countries and nutritional and economic factors have also been linked with the development of GERD and associated cancers (Jung et al., 2011). A number of these factors are given in Fig 7 as they ultimately reduce the acid production by stomach which on one hand increases intraabdominal pressure by poor digestion of food and bacterial dysbiosis and on the other hand impairs the antireflex barrier leading to onset of GERD. Moreover, Fig 7 also highlights certain other causative factors including medications, pregnancy, scleroderma, obesity and hiatal hernia.

\subsubsection{Diagnosis and Treatment}

According to Iwakiri et al., 2016, the diagnosis of GERD stems from presentation of symptoms. The most common method employed for diagnosing acid reflux is esophageal endoscopy. Asian countries use endoscopy regularly for diagnosing and conforming cases of GERD as this is a relatively cheap option in this region. The Western world uses methods, including endoscopy as well as pH-based tests to identify GERD since the disease is associated with lowering of $\mathrm{pH}$ in the esophageal region due to acid reflux. However, the $\mathrm{pH}$-based tests can sometimes lead to misdiagnosis as lowered $\mathrm{pH}$ is not exclusive to GERD and can be indicative of other complications and infections. Therefore, endoscopy is possibly a better method for correct diagnosis (Iwakiri et al., 2016).

The treatment options for GERD vary between proton pump inhibitors (PPI) and other drugs for suppressing acids in the data sets collected through artificial intelligence. However, these options are not found suitable for all patients and there is a need to move towards personalized medicine so as to satisfy patients having varying levels of medical needs. According to Vaezi et al., 2017, management of GERD has been associated with lifestyle changes and it has been found that management is a better option as compared to drug treatment in many cases. Also, it is important to note that patients suffering from GERD can present with varying symptoms and different levels of unease and pain, it is not possible to treat everyone and satisfy their needs with acid suppressors (Vaezi et al., 2017). According to Karim et al., 2011, some of the management approaches include lifestyle modifications. These modifications include scheduling meals properly and carefully, avoiding late dinners or foods rich in fats and oils. Also, walking after dinner has been noted as an important and effective measure for warding off severe acid refluxes. It is crucial to note that GERD is also aggravated through strenuous exercise and fatigue. Therefore, it is important that patients work alongside trainers and are properly guided as per what exercises they should follow to successfully manage their condition (Karim et al., 2011).

\subsubsection{Comparative analysis of Risk factors and Incidence of GERD (Gastroesophageal Reflux Disease) in between different cities of Pakistan}

Analysis of GERD prevalence among the Pakistani population has taken place from time to time through machine learning from previous records of different hospitals. A research conducted in 2005 with a study population of nine hundred and sixty-three individuals (aged above 15 years) reported that almost $24 \%$ of the population suffered from symptoms of GERD. Out of these $24 \%, 58 \%$ were males while $42 \%$ were females. Further analysis of these cases led to the identification of possible underlying causes revealed that most of these people never visited hospitals and relied on self-medication for treating the condition. Moreover, the instances of acid reflux were most commonly found associated with the use of spicy food (Jafri et al., 2005).

A study carried out in Abbottabad (Pakistan) linked GERD positively with Chronic Obstructive Pulmonary Disease (COPD). According to the findings of the study, it was evident that long term and aggressive cases of GERD, particularly in the old age population (people above the age of 60) led to severe bronchospasm, causing COPD. Furthermore, such conditions were found to be more common in males as compared to females (Khan et al., 2017). This has been backed with the ratios of smokers in the population. As smoking is a common cause of both, GERD and COPD, the presence of these conditions in males is linked with the data that there are higher number of male smokers in the Pakistani population as compared to females (Ahmed et al., 2008).

Analysis of the factors which contribute to the development of GERD showed obesity as an important factor. Studies from Pakistan as well as different parts of the world revealed that an increase in the body mass index could lead towards increase in the occurrence of GERD within a population (Zaman et al., 2016; Nadaleto et al., 2016). As an individual becomes obese, there is a significant increase in the gastrointestinal, cardiovascular and pulmonary disturbances, which can together lead towards different diseases including GERD (Nadaleto et al., 2016). All this data on disease prevalence based on different causative factors in Pakistani population is summarized in the Table 1.

In 2016, a survey was conducted for analyzing the understanding of physicians in Pakistan about the incidence and management of GERD. It was found that a great majority of the doctors inquired about heartburn and whether the situation developed after the intake of a meal. Only about half of the doctors involved in the study asked the patients whether they faced any difficulty in swallowing. This study concluded that since GERD can be presented with varying symptoms and the diagnosis could be confused with symptoms occurring due to different underlying conditions, it is important to work towards the development of a questionnaire, through data based on machine learning, which is specific to the Pakistani population and has been tested and validated in the same region. Some biologists are also working hard in order to compute data on different diseases using the approach of machine learning and artificial intelligence (Munawar et al ;Munawar et al., 2020; Munawar et al). Data collected through artificial intelligence is 
important to ascertain a diagnosis based on patient history and presenting symptoms (Shaukar et al., 2018). The use of innovative technology in biological sciences is also thought to be very efficient in the future studies (Munawar et al.; Munawar et al., 2020).

Table 1 Comparison of disease prevalence based on different causes

\begin{tabular}{|c|l|l|l|l|}
\hline \multicolumn{1}{|c|}{ Sr no. } & Self-medication for treating the condition, Spicy Food & $\begin{array}{l}\text { P8\% Male and 24\% } \\
\text { Female }\end{array}$ & Jafri et al., 2005 \\
\hline 1 & Long-term aggressive cases , smokers & $\begin{array}{l}\text { More prevalent in male } \\
\text { above } 60\end{array}$ & Khan et al., 2017 \\
\hline 2 & Obesity & $\begin{array}{l}\text { Prevalent in both male } \\
\text { and females }\end{array}$ & Nadaleto et al., 2016 \\
\hline 4 & Smoking & Prevalent more in males & Shaukar et al., 2018 \\
\hline
\end{tabular}

\section{Conclusion}

The worldwide prevalence of GERD and associated health complications, have made GERD to be a burden on healthcare systems. This study provides data on disease prevalence from different countries, along with data from Pakistani population. It throws light on some of the major disease risk factors and symptoms available from the literature by utilizing the concepts of artificial neural networks in this domain. Overall, data collected through artificial intelligence highlighted that GERD is a common disease with a worldwide prevalence and Asian countries are becoming increasingly more prone to this disease condition over the years. Pakistan being one of the Asian nations is no different in this regard. Clustered data sets and reports associating GERD with varying conditions have been reported from different regions of Pakistan over the years. The risk factors vary and there is a need to identify the trends of GERD generally found among the Pakistani population.

Therefore, it needs the attention of healthcare service providers and regulatory authorities to carefully manage the disease. It requires not only the preventive measures, but also the ground knowledge of disease prevalence, symptoms, and associated risk factors. A better diagnosis would only be possible if this epidemiological data would be made available and is critically analyzed to identify its relevance with other disease cases as well. This study, therefore, would assist in better diagnosing of GERD by carefully analyzing the risk factors, symptoms and prevalence of the disease. Moreover, identifying these factors would enable to educate and increase the awareness about GERD in population, which would have a better impact in controlling and monitoring the disease.

This study will also help us in identifying the risk of developing other diseases that are also associated with similar conditions. Therefore, both the diagnosis and treatment options in Pakistan need to be reviewed and revised with the concrete set of guidelines which are to be followed throughout the country. Analyzing the disease progression and risk factors from different cities and districts can help in ensuring that a meta-analysis-based research helps in adding to the demographic and epidemiological data related to the prevalence and complications of GERD among the Pakistani population. It is also important to work towards the development of a questionnaire in future researches, through data based on machine learning, which is specific to the Pakistani population and has been tested and validated in the same region. Through questionnaire testing and more validated comprehensive data can be collected in the future researches which in return help to predict, monitor and cure this disease more efficiently and thus, reducing the burden from economy.

\section{References}

[1] Ahmed, R., Rizwan-ur-Rashid, M. P., \& Ahmed, S. W. Prevalence of cigarette smoking among young adults in Pakistan. J Pak Med Assoc, 2008,. 58(11), 597-601.

[2] Argyrou, A., Legaki, E., Koutserimpas, C., Gazouli, M., Papaconstantinou, I., Gkiokas, G., \& Karamanolis, G. Risk factors for gastroesophageal reflux disease and analysis of genetic contributors. World journal of clinical cases, 2018, 6(8), 176.

[3] Fujiwara, Y., Machida, A., Watanabe, Y., Shiba, M., Tominaga, K., Watanabe, T. \& Arakawa, T. Association between dinnerto-bed time and gastro-esophageal reflux disease. The American journal of gastroenterology, 2005, 100(12), 2633.

[4] Furuta, T., Sugimoto, M., Kodaira, C., Nishino, M., Yamade, M., Ikuma, M. \& Hishida, A.. CYP2C19 genotype is associated with symptomatic recurrence of GERD during maintenance therapy with low-dose lansoprazole. European journal of clinical pharmacology, 2009, 65(7), 693-698.

[5] Herbella, F. A., \& Patti, M. G. Gastroesophageal reflux disease: From pathophysiology to treatment. World journal of gastroenterology: WJG, 2010, 16(30), 3745.

[6] Iwakiri, K., Kinoshita, Y., Habu, Y., Oshima, T., Manabe, N., Fujiwara, Y. \& Ashida, K. Evidence-based clinical practice guidelines for gastroesophageal reflux disease 2015. Journal of gastroenterology, 2016, 51(8), 751-767. 
[7] Jafri, N., Jafri, W., Yakoob, J., Islam, M., Manzoor, S., Jalil, A., \& Hashmi, F.. Perception of gastroesophageal reflux disease in urban population in Pakistan. Journal of the College of Physicians and Surgeons--Pakistan: JCPSP, 2005, 15(9), 532-534.

[8] Jung, H. K. Epidemiology of gastroesophageal reflux disease in Asia: a systematic review. Journal of neurogastroenterology and motility, 2011, 17(1), 14.

[9] Karim, S., Jafri, W., Faryal, A., Majid, S., Salih, M., Jafri, F. \& Tariq, U. Regular post dinner walk; can be a useful lifestyle modification for gastroesophageal reflux. Journal of the Pakistan Medical Association, 2011, 61(6), 526.

[10] Khan, H. N., Suleman, A., Ullah, R., Abdullah, A., \& Naz, S. GASTRO ESOPHAGEAL REFLUX DISEASES IN CHRONIC OBSTRUCTIVE PULMONARY DISEASE PATIENTS. Journal of Ayub Medical College Abbottabad, 2017, 30(1), 64-66.

[11] Mao, W. M., Zheng, W. H., \& Ling, Z. Q. Epidemiologic risk factors for esophageal cancer development. Asian Pac J Cancer Prev, 2011, 12(10), 2461-2466.

[12] Menezes, M. A., \& Herbella, F. A. Pathophysiology of gastroesophageal reflux disease. World journal of surgery, 2017, 41(7), 1666-1671.

[13] Munawar HS, Hammad A, Ullah F, Ali TH. After the flood: A novel application of image processing and machine learning for post-flood disaster management. InProceedings of the 2nd International Conference on Sustainable Development in Civil Engineering (ICSDC 2019), Jamshoro Pakistan 2019a Dec (pp. 5-7).

[14] Munawar, H. S. An Overview of Reconfigurable Antennas for Wireless Body Area Networks and Possible Future Prospects. International Journal of Wireless and Microwave Technologies (IJWMT);2020, 10(4), 1-8.

[15] Munawar, H. S. Applications of Leaky-wave Antennas: A Review. International Journal of Wireless and Microwave Technologies (IJWMT); 2020, 10(4), 56-62.

[16] Munawar, H. S. Reconfigurable Origami Antennas: A Review of the Existing Technology and its Future Prospects. International Journal of Wireless and Microwave Technologies (IJWMT); 2020, 4, 34-38.

[17] Munawar, H. S., \& Maqsood, A., Mustansar, Z. Isotropic Surround Suppression based Linear Target Detection using Hough Transform. International Journal of Advanced And Applied Sciences; 2017, 4(8), 37-42.

[18] Munawar, H. S., Khalid, U., Jilani, R., \& Maqsood, A. (2017). Version Management by Time Based Approach in Modern Era. International Journal of Education and Management Engineering, 4, 13-20.

[19] Munawar, H. S., Qayyum, S., Ullah, F., \& Sepasgozar, S. Big Data and Its Applications in Smart Real Estate and the Disaster Management Life Cycle: A Systematic Analysis. Big Data and Cognitive Computing; 2020, 4(2).

[20] Munawar, H. S., Zhang, J., Li, H., Mo, D., \& Chang, L. Mining multispectral aerial images for automatic detection of strategic bridge locations for disaster relief missions. In Pacific-Asia Conference on Knowledge Discovery and Data Mining (pp. 189200). Springer, Cham. . (2019b, April)

[21] Munawar, H. S., Khalid, U., \& Maqsood, A. Fire detection through Image Processing; A brief overview.

[22] Munawar, H. S., Khalid, U., \& Maqsood, A. Modern day detection of Mines; Using the Vehicle Based

[23] Detection Robot. Munawar HS, Awan AA, Maqsood A, Khalid U. REINVENTING RADIOLOGY IN MODERN ERA.

[24] Munawar, H. S. Flood Disaster Management: Risks, Technologies, and Future Directions. Machine Vision Inspection Systems: Image Processing, Concepts, Methodologies and Applications, 2020, 1, 115-146.

[25] Munawar, H. S. Image and Video Processing for Defect Detection in Key Infrastructure. Machine Vision Inspection Systems: Image Processing, Concepts, Methodologies and Applications, 2020, 1, 159-177.

[26] Nadaleto, B. F., Herbella, F. A., \& Patti, M. G.. Gastroesophageal reflux disease in the obese: Pathophysiology and treatment. Surgery, 2016, 159(2), 475-486.

[27] Shaukat, F., Jawaid, M., Rizwan, A., \& Kumar, B. Primary-care physicians' perceptions and practices regarding History of Gastro Esophageal Reflux Disease: a national survey. Rawal Medical Journal, 2018, 43(4), 764-766.

[28] Vaezi, M. F., Pandolfino, J. E., Vela, M. F., \& Shaheen, N. J.. White paper AGA: Optimal strategies to define and diagnose gastroesophageal reflux disease. Clinical Gastroenterology and Hepatology, 2017, 15(8), 1162-1172.

[29] Veugelers, P. J., Porter, G. A., Guernsey, D. L., \& Casson, A. G. Obesity and lifestyle risk factors for gastroesophageal reflux disease, Barrett esophagus and esophageal adenocarcinoma. Diseases of the Esophagus, 2006, 19(5), 321-328.

[30] Zaman, H., Zeb, J., Farooq, M. U., Shah, M. T., Qayyum, S. W., \& Abbasi, M. A. SOCIO-DEMOGRAPHIC CHARACTERISTICS OF GASTROESOPHAGEAL REFLUX DISEASE PATIENTS. Pakistan Journal of Physiology, 2016, 12(3), 40-43.

\section{Author's Profile}

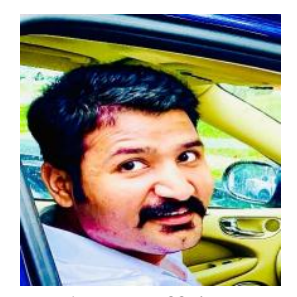

regulatory affairs.
Mustafa Pasha is an independent consultant on computational modelling and simulation on medical and health related topics. He has a master's in computational sciences and engineering from NUST, Pakistan and a $\mathrm{PhD}$ in Applied computing from Lincoln, New Zealand. He has a dedicated set of expertise in drug design and discovery, his past work includes work on cancer cell proliferation and human cell cycle modelling and simulation. Besides his research profile, he has number of achievements in health business, procurement, and novel solutions consultations. He holds the privilege to be nominated in Canterbury Business Champion, New Zealand. His research interests include, Pharmaceutical Formulations, Intelligent Modelling and Simulation, Artificial intelligence, Machine learning, Data Analysis, Industrial Business Consultancy and 
How to cite this paper: Mustafa Kamal Pasha, " Machine Learning and Artificial Intelligence Based Identification of Risk Factors and Incidence of Gastroesophageal Reflux Disease in Pakistan", International Journal of Education and Management Engineering (IJEME), Vol.11, No.5, pp. 23-31, 2021. DOI: 10.5815/ijeme.2021.05.03 